\title{
PAIRS 2018 Keynote Lecture: The Next Decade in Interventional Radiology: Do We Need More Disruptive Innovation or Better Integration?
}

Interventional radiology (IR) has matured from a renegade disruptive subgroup of diagnostic radiologists to a recognized subspecialty (and in some countries, a primary specialty). This lecture reviews two major theories of change and competition from the perspective of IR and then explores some of the critical challenges ahead of us. The central role of innovation in the past, present, and future of IR is highlighted. The future potential for IR may never have been as great as it is today.

I am deeply honored to be giving the PAIRS 2018 Keynote Lecture. IR is growing all over the world and, in these uncertain times, it is a wonderful opportunity for all of us to come together as one and focus on a common future, that of our specialty. The title of this lecture asks a very complicated question, so in order to answer it, we will first look at a few of the business concepts that have been applied to medical specialties. We will then explore some of the issues that we need to address as a specialty as we move forward.

The concept of disruptive innovation was proposed in 1995 by Clayton Christensen of Harvard Business School. The concept is an attempt to describe the interactions between companies and consumers, particularly when there are rapid changes in the environment. There have been several modifications, but "Disruptive" is one of the most popular terms utilized today when describing changes in the business world. This figure is the classic graphic representation of disruptive innovation, with the white lines representing the consumers and the dashed lines representing the businesses or organizations. As the incumbent businesses chase the high-end consumers, it leaves a gap where the low-end consumers are not served and the mainstream consumers are undeserved. This provides an opportunity for disruptive innovation. What is a "disruptive innovation"? This is something that is less costly and of lower quality than needed for high-end consumers; in other words, the target is the low-end consumers. Disruptive innovations do improve over time, but they still retain their cost advantage and are often overlooked by industry that is focused on a high-end consumer. Only after the disruptive innovation captures mainstream consumers, does it gain the attention of the dominant industry entities. The original disruptive innovation theory often required that new business models be part of the process that the process took time and it was not necessarily the technology that was disruptive, but rather what was done with it. Most importantly, something could be disruptive but not necessarily successful. There have been a couple of refinements to the theory. Now, it is known that the customers drive change and disruption drives everybody up market, meaning as the disruptor chases the incumbent, the incumbent works harder to stay ahead. There is another form of disruption called "new market disruption," in which a technology, organization, or business reaches a whole new set of customers rather than just the low-end customers. In this scenario, a group that was previously being completely ignored has now participating in the market.

Now, IR likes to think of itself as disruptive and its innovations as disruptive. For example, think about long-term patency of peripheral vascular interventions. Surgery is continually seeking to find the longest patencies possible and ignoring interventions that have short-term patency. Catheter-based interventions, which were initially relatively inexpensive and had a low burden upon the patient, focused initially on short-term patency. But, we too are now seeking the long-term patencies for our interventions; in other words, we are moving our interventions upmarket.

Angioplasty did more than disrupt surgical bypass. Remember that our specialty roots are in invasive diagnosis as part of diagnostic radiology. So, in 1964, Charles Dotter disrupted diagnostic radiology and in addition to other specialties when he did his first angioplasty.

Now, I would like to discuss a different framework for thinking about our environment. Another Harvard business school professor, Michael Porter, proposed a theory of competitive forces in 1979. This is a means of organizing thinking of how competitors not only interact with each other, but are also impacted by external forces.

First, let us define a competitor as somebody who does what you do. In 1964, when interventional radiologists (IRS) were just differentiating themselves, their competitors were radiologists. Few other people had access to imaging equipment. In 2018, the list is much longer and not only does it include diagnostic radiologists, but also a large number of nonradiologic specialists. Now, how do competitors change you? Well, they can certainly just outnumber you, but they may provide better service or a different quality of service - potentially better. They may cost less and they may be able to make strategic alignments that influence how you can practice.

One of the groups that has influence on competitors is the buyers, the people who utilize your services. The largest segments of this group are patients and other physicians, but buyers also include hospitals, businesses, insurance, the government, and anybody else that utilizes you or your services. Buyers change you because they can shift work to 
your competitors. They will seek out alternative, less costly treatments that you have to offer; they may decide they want you to do the work but pay you less, or they may just buy you outright. In fact, in the United States, this is the trend with most physicians now working for hospitals.

Next, let us talk about suppliers. Suppliers provide not just materials or objects but anything that is needed for you to be successful. I would argue that probably the most important supplier for us right now are medical schools. Medical students are the individuals that are just coming into our specialty and will have the longest opportunity to advance IR. Other suppliers are industries which provide us tools, science and engineering which provide us with new ideas and new technology and venture capital to fund all of these. How can suppliers change you? They can bring you new skills and technologies to allow you to perform new procedures. However, the cost of supplies can really change your utilization and, in the end, any of your suppliers can also supply your competitors. A typical example of this are the hybrid operating rooms that are now ubiquitous throughout the world.

Let us now talk about new entrants. These are people or things that begin to do part of what you do. Now, this could be another physicians or other kind of providers, but keep in mind that this does not have to be a living thing. New entrants can be software or even hardware. New entrants can dramatically change your world. They can change the value of some or all of your skills and force you to restructure or refocus your practice to accommodate them. Over time, they can become substituters, a group that we will discuss in the next paragraph. A current example is the increasing number of physician assistants and advanced practitioners relative to the number of independent physicians in the United States. Another example is the potential combination of angiographic guidance software and robotics that could automate selective catheterization of the hepatic arteries.

Now, let us move to the last group - the substituters. These are the people, the things, and the organizations that achieve the same outcomes that you have worked so hard to be able to achieve. They are often faster, safer, better, or cheaper the way we like to describe ourselves. Moreover, they do not need to disrupt to substitute and, although we think IR as a classic disruptor, IR is a classic substituter. For example, let us look at decompression of the portal vein in patients with severe portal hypertension. Percutaneous image-guided transjugular portosystemic shunt has essentially replaced surgical shunts, with the average graduating surgical residents having participated in 0.1 shunts in their 5 years of training.

Having explored some basic business concepts from an IR point of view, consider that there are other ways to think about IR. Specialties like everything else have a life cycle, and it is interesting to line this up with the elements of the life cycle of human beings. The basic phases of a life cycle are the newborn who has unknown potential and is very dependent; the adolescent and young adult who is changing rapidly, seeks independence and, in fact, is rebellious; those in middle age who begin to crave to stability and like incremental change; and lastly, those in old age, who often are resistant to change and focused on self-preservation.

Let us briefly look at this applied to IR. When IR was in its infancy, there was no structure, there was no identity, and no one knew where it was going to turn into. Only the most basic tools were available. When the physicians saw a problem, they immediately created their own solution and then used it in practice, creating a culture of physician innovation that has permeated the specialty since then. This was also an era of minimal regulation. Consider this image of Charles Dotter and Bill Cook meeting at the Radiological Society of North America in the 1960s. There is a blowtorch sitting on the table between them used to shape catheters in the meeting hall. Can you imagine bringing a blowtorch into a meeting venue or your angio suite today?

What was adolescent IR? I think it was the rise of the subspecialty with societies, journals, and meetings appearing all over the world. Fellowships appeared and then became organized, leading to certification (in other words standardization of the educational process in the output). IR relished its maverick, daring, rebellious reputation. During this period, there was an increasing focus on intervention, and the device industry grew so that it could supply us with the things that previously we had to make on our own. However, this also brought with it conflict with other specialties as they began to observe the successes of IR. In many instances, we became obsessed with warding off competitors and developed a sense of entitlement for the procedures that we had developed.

Now, we arrive at the middle age of IR. We have achieved specialty status in certain areas and fellowships have now become residencies in some areas of the world. In a very real sense, IR has joined the medical establishment as we have structured ourselves to fit in with the rest of medicine. However, with this maturation comes slower innovation and mostly incremental changes - stents that last a little bit longer and balloons that are a little stronger or give off a drug. However, in a very positive change, we are now focused on competing rather than engaging in conflict. We now understand the importance of nonprocedural care to the future of the specialty and the care of our patients, and we understand the importance of becoming a content expert in addition to being a technical expert. However, we live in a world of intense regulation - government regulation of practice in industry and local regulation of practice. There is now intense pressure to reduce the cost 
of everything we do, which limits some of our ability to provide interventions.

I have to admit that I do not like talking about the old age of IR as much as the other phases maybe because I have gray hair. However, unlike you and me, I believe that limitations in old age are avoidable for IR if we focus on that intrinsic quality of the specialty - innovation. We must adapt the ways that we innovate to the new environment, but if we can figure out how to do this, I believe that IR will be able to stay young essentially forever.

How do we keep innovating in IR as the world changes around us? Let us talk for a moment about directionality in innovation. Currently, many devices are first developed on the bench and then moved to the bedside; when this happens, innovation becomes a driver of clinical need. All device developments require a lot of upfront investment to get to the clinical realm, so there is an incentive to support the existing technology. We have all seen devices that we look at and wonder how they got so far, often because there has been so much investment that industry is reluctant to let them fade away. In the future, we need to return to our roots where a clinical need observed at the bedside then drives innovation. However, the way that we identify these needs has to be different. We need a full understanding of the need rather than focusing on the immediate problem in front of us. This involves bringing in the entire stakeholder set as we move this forward. This includes the patient, other physicians, insurers, regulators, and anybody who might be touched by this innovation. The likelihood of success of an innovation is much higher when the input and perspectives of the key stakeholders have been incorporated into the design at the beginning.

Innovation is a cycle, and at the top of the cycle is the unmet clinical need. This is often something that may not be apparent to those who are working every day and have developed work around or other compensatory mechanisms. However, once an unmet need is identified, it drives the invention and the design, the prototyping, and all of the other steps. Keep in mind that an unmet clinical need that you encounter once a year, no matter how irksome to you, is unlikely to be successful in all the steps of the innovation process. The unmet clinical needs that you encounter five, six, and seven times a day are the ones that ultimately will make through the full cycle of innovation and turn into a product.

I propose that in the future we should have different targets for our innovations. In the past and often still in the present, we focus on established pathology - the blocked artery, the bleeding artery, the tumor, and the infected space. What we do is utilize catheters, needles, guidewires, and imaging to devise a solution that replaces an existing surgical procedure. In the future, we need to think about intervening earlier in the disease process, perhaps even before established pathology has developed. We also need to think about patient-centered outcomes. Measurement of quantitative success of specific intervention is just one aspect of determining outcomes. The patient's perception of the outcome is probably more relevant. Think about target lesion revascularization as an outcome for peripheral arterial intervention compared to patient satisfaction with their ability to walk. We also need interventions that challenge the current paradigms. If we only allow ourselves to think about the disease processes in the framework of the current paradigms, we will be destined to reproduce the surgical procedures that have developed to treat these conditions. If we can think in new ways about diseases, we may find new approaches and create new markets. We need to start working on diseases that previously were considered untreatable. For example, lymphatic diseases for which we are now beginning to develop some very successful interventions.

To illustrate this, let us play with an idea for a minute. What if there was an IR treatment for diabetes. Something that would normalize serum glucose for some period of time, say 10 years. Well, there are 30 million diabetics in the United States and 84 million prediabetics with a total treatment cost of 258 billion dollars per year. Think about the impact on the cost of care and the improved longevity and quality of life that this kind of intervention could potentially bring. So, how do we get to this magical place of creating interventions that really alter the course of disease? We need to change our practice, our education, our research, and how we collaborate. So, let us talk about IR practice.

Today, by and large, we are still considered procedural experts; however, tomorrow, we need to be content experts. We need to know as much about the diseases we treat as the nonproceduralists who also care for these patients. We have to be full-fledged members of multidisciplinary care teams so that we are on an equal footing with the other services taking care for these patients. This means that we are in the clinics, we are using the electronic medical records, we are attending conferences and, most importantly, we are getting early exposure to patients. We are not waiting until patients develop a pathology and someone decides to look for a less invasive way to treat it. We need early exposure to the management of these patients to understand where the challenges are that will lead to new targets for our interventions.

Perhaps not as influential on innovation but germaine to clinical practice is the concept of IR is an essential service. IR is like the Intensive Care Unit, the anesthesia service, and the emergency room. A large hospital just cannot function without it. The faster we provide our services, the 
faster the patient would get out of the hospital. We have found in our own internal studies that there is a one-to-one correlation with the delay in providing an inpatient interventional service and extensions of the length of stay. We need a new IR subspecialty emergency general IR, or acute care IR. For large institutions, this would provide 24/7, 365 in-house coverage. The decreased length of stay would fund this service and, in an important way, addresses one of the features of competition that we talked about earlier. One of the reasons why trauma surgeons are beginning to perform embolization is that IR is not right there when that service is needed. So, think of the power of being present all the time.

How we educate IRS is going to have a huge impact on our future. We are achieving great standardization in the educational process and we are moving education into the medical schools. All of these are critical to the future of IR and the provision of image-guided intervention to our patients. IR per se is becoming a base specialty and we need to allow for the evolution of subspecialties of IR over time. Most importantly, we need to include innovation and research in our educational process. We cannot focus just on producing excellent technicians, we need to produce people who are clinician-scientists. We also need to understand that if image-guided interventions are really as beneficial as we believe, then we need to get them into practice as broadly as possible. In some circumstances, this will mean embracing the idea of training non-MDs to deliver some of our services.

The process of education is going to change dramatically as well. Simulations are going to grow an importance, not just procedural simulations, but patient management simulation as well. The idea that the first time you do some high-risk new intervention is on a living human being is going to gradually fade away as the simulators grow in fidelity and sophistication. The process of transfer of knowledge is changing. Keep your eye on the HBX experiment of Harvard Business School, in which people from all over the world are in live video conference with the faculty member and each other. As HBX has evolved, the faculty have started providing content and allowing learners to actually teach themselves. We need to value IR research as much as we do technical prowess. We need to develop multidimensional devices that not only support structures and sense what is going on, but can communicate with the outside world and administer treatments. We need to focus on the disease, not the pathology. This is where being a content expert, having a much deeper understanding of the disease processes that we are dealing with becomes critically important, and why valuing IR research is so fundamental.
I think that everyone would agree that it is very difficult to study interventions in the current world. The traditional randomized controlled trial is becoming less and less practical. The patient populations are too highly selective compared to day-to-day practice, and the technologies change so fast that long studies can become outdated before they conclude. There is also a huge variability in the operators, something that has not readily discussed but has a huge impact on the outcomes. Alternatives include studying free-living cohorts, looking at the whole populations and their response to intervention, or focusing on patient-centered outcomes.

IR has traditionally been a kind of a loner specialty in terms of innovation - we have been off on the side, working in the dark, coming up with solutions in our garages and on our kitchen tables. This is not the way of the future. Collaboration is the way of the future. Terry Tao PhD won the Fields Medal in 2006 (the Nobel Prize equivalent for young mathematicians). Based on a cooperative problem-solving approach that included more than thirty collaborators; not just mathematicians, but across disciplines, across societal boundaries, and across geographies. Without collaboration, IR will not be able to take advantage of the incredible knowledge that exists among the other disciplines. Consider the power of bringing proteomics and genomics into IR and collaborating with individuals who really understand biomaterials and metals in a far more sophisticated way than we do now. We could apply remote sensing, devices that can communicate with the outside world, theranostics, and population science to help us target those disease processes where we can have the most impact. I think it is really true that at this point we do not know what we do not know.

Imagine the ability to draw a sample of blood from an individual that allows creation of a truly biocompatible device containing a specific medication needed by the patient, has the ability to transmit data, and can be sterile printed right in your lab before placing into the patient. This would allow infinite variability of devices without maintaining shelf stock, and devices that are truly patient specific. However, without collaboration across all the many basic science and bioengineering disciplines, we will never get to this point. We will remain stuck in the world of premanufactured limited function devices that we have now.

In conclusion, I hope I have convinced you that there is no one theory or framework that neatly captures IR. However, innovation is the constant, the defining feature of our specialty. How we do innovate though, will have to change as the world around us changes. So, whether you 
think of us as disruptive, integrative, collaborative, or as a substituter, I think it is all good - because we are all that!

Thank you very much, it has been my privilege to speak to you today, and I hope you enjoy the rest of the meeting.

John A. Kaufman ${ }^{1,2}$

${ }^{I}$ Department of Interventional Radiology, ${ }^{2}$ Dotter Interventional Institute, Oregon Health and Science University, Portland, Oregon 97239, USA. E-mail:kaufmajo@ohsu.edu
This is an open access journal, and articles are distributed under the terms of the Creative Commons Attribution-NonCommercial-ShareAlike 4.0 License, which allows others to remix, tweak, and build upon the work non-commercially, as long as appropriate credit is given and the new creations are licensed under the identical terms.

\begin{tabular}{|l|l|}
\hline \multicolumn{2}{|c|}{ Access this article online } \\
\hline Quick Response Code: & Website: \\
& www.arabjir.com \\
\cline { 2 - 2 } & DOI: \\
\hline
\end{tabular}

How to cite this article: Kaufman JA. PAIRS 2018 keynote lecture: The next decade in interventional radiology: Do we need more disruptive innovation or better integration?. Arab J Intervent Radiol 2018;2:41-5. 